ARTICLE

Received 3 Oct 2016 | Accepted 24 Feb 2017 | Published 13 Apr $2017 \quad$ DOl: 10.1038/ncomms15063 OPEN

\title{
Local inhibition of microtubule dynamics by dynein is required for neuronal cargo distribution
}

Shaul Yogev' ${ }^{1}$, Celine I. Maeder ${ }^{1}$, Roshni Cooper ${ }^{2}$, Mark Horowitz $^{3}$, Adam G. Hendricks $^{4}$ \& Kang Shen ${ }^{1}$

Abnormal axonal transport is associated with neuronal disease. We identified a role for DHC-1, the C. elegans dynein heavy chain, in maintaining neuronal cargo distribution. Surprisingly, this does not involve dynein's role as a retrograde motor in cargo transport, hinging instead on its ability to inhibit microtubule (MT) dynamics. Neuronal MTs are highly static, yet the mechanisms and functional significance of this property are not well understood. In disease-mimicking dhc-1 alleles, excessive MT growth and collapse occur at the dendrite tip, resulting in the formation of aberrant MT loops. These unstable MTs act as cargo traps, leading to ectopic accumulations of cargo and reduced availability of cargo at normal locations. Our data suggest that an anchored dynein pool interacts with plus-end-out MTs to stabilize MTs and allow efficient retrograde transport. These results identify functional significance for neuronal MT stability and suggest a mechanism for cellular dysfunction in dynein-linked disease.

\footnotetext{
${ }^{1}$ Department of Biology, Howard Hughes Medical Institute, Stanford University, 385 Serra Mall, California 94305, USA. ${ }^{2}$ Department of Electrical Engineering, Stanford University, 350 Serra Mall, California 94305, USA. ${ }^{3}$ Department of Electrical Engineering and Computer Science, Stanford University, 353 Serra Mall, California 94305, USA. ${ }^{~}$ Department of Bioengineering, McGill University, 817 Sherbrooke Street West, Montreal, Quebec, Canada H3A 0C3. Correspondence and requests for materials should be addressed to K.S. (kangshen@stanford.edu).
} 
$\mathrm{T}$ he neuronal microtubule (MT) cytoskeleton provides structural support and serves as tracks for cargo transport. The large size of neurons necessitates unique properties for their MTs, which is reflected by neuron-specific MT-associated proteins and post-translational modifications ${ }^{1-4}$. The importance of functional MTs to the nervous system is underscored by neurodegenerative conditions associated with mutations in cytoskeletal components ${ }^{5,6}$.

One defining property of a MT polymer is its dynamic instability. In vitro, MTs frequently undergo transition from growth to shrinkage (catastrophe) and vice versa (rescue). In vivo, MT dynamics are regulated by a large number of cofactors that can anchor minus ends, promote polymerization or capture the growing end ${ }^{7-10}$.

Neuronal MTs are significantly less dynamic than MTs in most cell types, and they are more resistant to depolymerization by cold or Nocodazole ${ }^{11,12}$. In the axon, cold or drug-resistant MT seeds extend labile fragments ${ }^{11,13-15}$. Axonal MT dynamics reflect the interplay between stabilizing MAPs and tubulin polyamination and destabilizing factors such as depolymerizing kinesins, severing enzymes and Stathmins $1,3,4,16-18$. We recently developed a method that allows examining how this interplay shapes steady-state polymer length, abundance and distribution ${ }^{19}$. MT dynamics in the dendrite are less well understood. MAP2 likely plays a key role, but the knockout mice only exhibit a moderate reduction in MT density, suggesting the existence of additional mechanisms ${ }^{20,21}$. Importantly, the in vivo consequences of a loss of neuronal MT stability have not been explored.

Cytoplasmic dynein is the main minus-end-directed motor in eukaryotic cells. A heavy chain (DHC) dimer provides MT binding and energy production, and its activity and cargo specificity are regulated by a host of co-factors ${ }^{22-24}$. Dynein's most established role in neurons is in retrograde axonal transport, and impaired function of the heavy chain or other subunits leads to cargo trafficking defects ${ }^{25-29}$.

Mutations in human DHC (DYNC1H1) or accessory proteins lead to several symptoms. In particular, an N-terminal region, which mediates dimerization and assembly of the complex, seems to be a hotspot for mutations that result in spinal muscular atrophy (SMA) $)^{30-34}$.

In addition to transporting cargoes, immobilized dynein can move MTs, either in neurons or in non-neuronal cells. Studies in cultured sympathetic neurons implicated this function in populating the growing axon with plus-end-out MTs and in resisting contractile forces ${ }^{35-37}$. In cultured fly neurons dynein moves minus-end-out MTs out of the developing axon ${ }^{38}$.

In non-neuronal cells, anchored dynein can also serve as a plus-end tether ${ }^{8,39-42}$. In vitro, growing MTs buckle or shrink upon reaching a barrier, but when the barrier is coated with dynein, the MT filament straightens under tension induced by the motor $^{43,44}$. These results suggest that anchored dynein can tether and stabilize MTs by interacting with their tips. Recently, MT tethering by dynein was implicated in synapse stabilization in cultured cortical neurons ${ }^{41}$.

Here we identified a role for DHC-1, the Caenorhabditis elegans dynein heavy chain, in maintaining the correct distribution of neuronal cargo. In $d h c-1$ mutants, cargo accumulates at the tip of the dendrite and is concomitantly reduced at normal locations. Surprisingly, defective minus-end-directed transport is not driving this phenotype. Our data suggest that DHC-1 regulates the spatial distribution of dendritic MTs and suppresses MT dynamics. The latter function is required locally, at the tip of the dendrite, where excessively dynamic MTs buckle, twist and loop in $d h c-1$ mutants. These unstable MTs trap cargo, leading to the ectopic accumulations and reduced availability of cargo at endogenous sites. We propose that anchored dynein tethers MT plus-ends at the dendrite tip to ensure correct cargo distribution. A similar mechanism may underlie some of the cellular defects that occur during dynein-related disease, as cargo trapping by unstable MTs is observed in a SMA-mimicking $d h c-1$ allele.

\section{Results}

wy743 mutants display ectopic cargo accumulations. The C. elegans cholinergic motor neuron DA9 sends out an anteriorly directed dendrite and a posterior axon that extends dorsally to join the dorsal nerve cord. A string of en-passant presynapses form in a restricted region of the axon (Fig. 1a). In wildtype (wt) L4 larvae, synaptic vesicle precursors (SVPs), labelled by synaptogyrin/SNG-1::GFP, concentrate at the presynaptic domain. SVPs are transported throughout the dendrite and distal axon but do not accumulate there ${ }^{45}$ (Fig. 1b).

From an unbiased forward genetic screen we isolated a mutant, wy743, with an abnormal distribution of synaptogyrin/SNG$1::$ GFP in DA9. Two ectopic accumulations of synaptogyrin/ SNG-1::GFP puncta are the defining feature of the wy743 phenotype: one at the tip of the axon, and a second accumulation at the tip of the dendrite (Fig. 1c-e). In addition, dendrite length is reduced in wy743 mutants to about $63 \%$ that of wt (Fig. 1f). SVP accumulations were also observed with another SVP marker, GFP::RAB-3 (Supplementary Fig. 1). The wy743 phenotype is also manifested in other parts of the nervous system, including the DB neurons (Supplementary Fig. 2).

To learn whether the wy743 defects are restricted to SVPs, we examined the localization of mitochondria. In wt controls, the mitochondrial marker TOMM-20::YFP is evenly distributed into small puncta along the dendrite (Fig. 1g). These puncta are displaced to the tip of the dendrite in wy743 mutants, leading to an ectopic accumulation (Fig. 1h). We conclude that wy743 affects different types of neuronal cargoes in several classes of C. elegans neurons.

To test whether the axonal SVP accumulations represent cargo sorting defects, we generated wy743; unc-104(e1265) double mutants. In unc-104 mutants SVPs are depleted from the axon ${ }^{46}$ (Supplementary Fig. 1E). No axonal SVPs were detected in wy743; unc-104(e1265) double mutants (Supplementary Fig. $1 \mathrm{~F}, \mathrm{H})$, suggesting that axonal cargo is not mis-sorted in wy743 mutants. Since SVPs are already abundant in the dendrite of unc-104 single mutants ${ }^{46}$ (Supplementary Fig. 1E), it is not possible to conclude from this experiment whether dendritic SVP accumulations in wy743 are due to sorting defects in dendrites. Instead, we conducted an analogous experiment with the mitochondrial marker TOMM-20::YFP. We found that the TRAK/Milton homologue, trak-1, is required for mitochondria to enter dendrites (Fig. 1i). In wy743; trak-1(tm1572) double mutants the TOMM-20::YFP signal was completely eliminated from the dendrite (Fig. 1j). These results suggest that neither the axonal nor the dendritic accumulations in wy743 mutants occur as a result of cargo missorting.

$w y 743$ is an allele of dynein heavy chain $\boldsymbol{d h c}$-1. We mapped wy743 to two substitutions in conserved residues of DHC-1. M563T affects the N-tail domain, and R1782C is located in the linker domain (Fig. 2a,b). A M582L mutation in human DYNC1H1 leads to dominant SMA ${ }^{32,33}$. To gain insight into the cellular mechanisms that may be involved in SMA we engineered the mutation into C. elegans dhc-1 using CRISPR-Cas9 (ref. 47). The allele, designated $d h c-1(S M A)$, resulted in a similar phenotype to $w y 743$ (Fig. 2c). To further test the link between C. elegans dhc-1 mutations and neuronal dysfunction in other systems, we generated a worm $L O A$ allele ${ }^{48}$. The mouse $L O A$ 
mutation reduces dynein complex assembly and affinity to MTs during ATP hydrolysis ${ }^{34,49}$. dhc-1(LOA) worms showed cargo accumulations reminiscent of the SMA and wy743 alleles (Fig. 2c).

To probe the mechanism leading to neuronal disease and to the phenotypes we observe in DA9, we engineered the SMA mutation into a rat DHC1-FLAG construct ${ }^{50}$, immunoprecipitated dynein from COS-7 cells onto beads and performed force measurements using an optical trap. Interestingly, although mutant and wt dynein produced similar stall forces, processivity under load was reduced in the mutant (Fig. $2 \mathrm{~h}-\mathrm{j}$ ). Speed was not significantly affected $\left(556 \pm 436 \mathrm{~nm} \mathrm{~s}^{-1}\right.$ for wt, $491 \pm 314 \mathrm{~nm} \mathrm{~s}^{-1}$ for mutant, $P=0.06$ ). Consistently, the LOA allele also exhibits defects in processivity but not force generation ${ }^{49}$.

Four lines of evidence suggest that wy743 impairs DHC-1 function. First, axonal SVP accumulation has been previously attributed to reduced dynein function in C. elegans ${ }^{27}$. Second, the $L O A$ and SMA mutations phenocopy wy743 in vivo (Fig. 2c), and
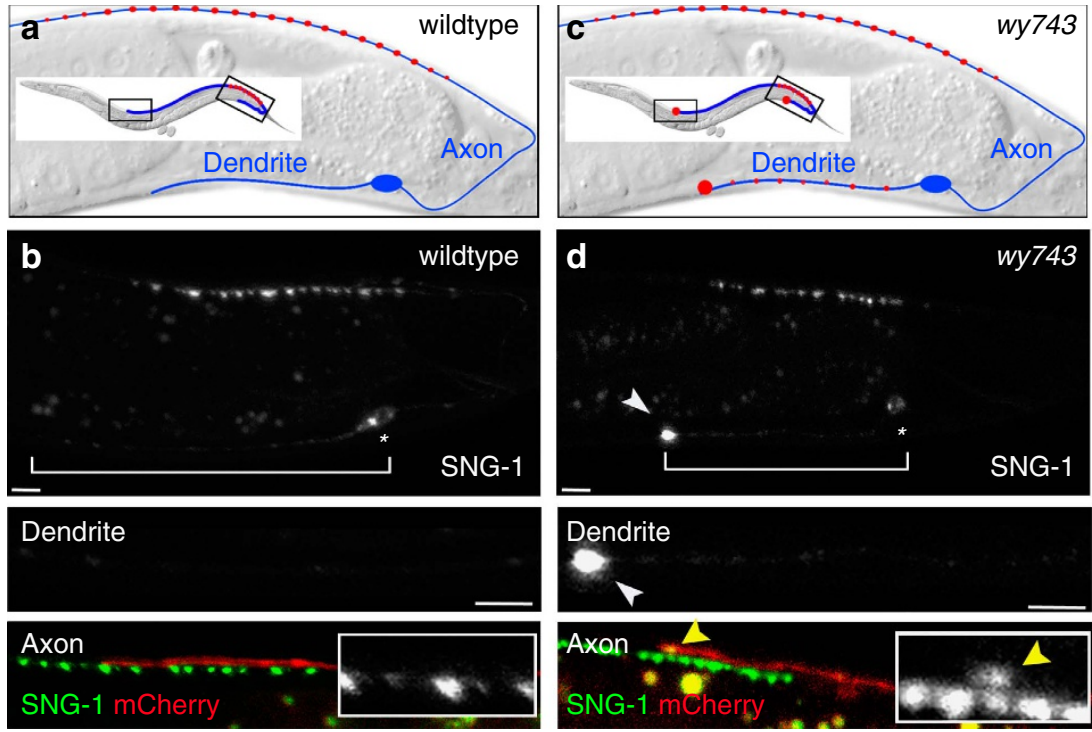

e

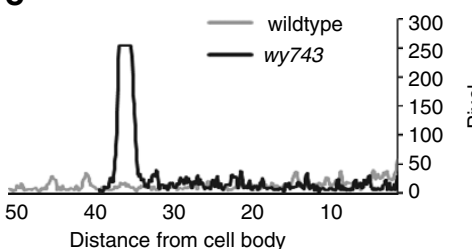

$f$

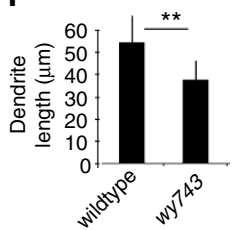

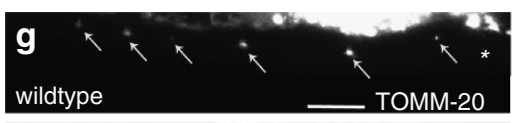
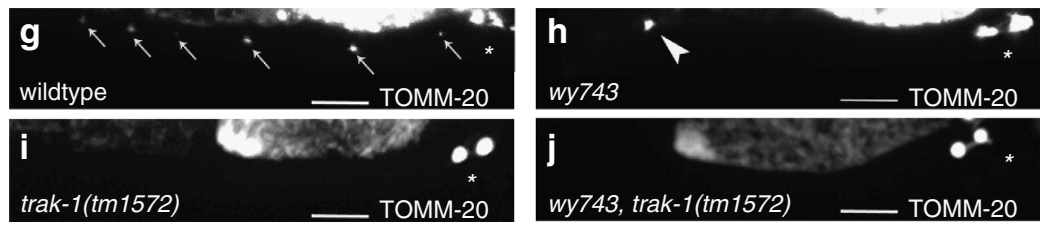

Figure 1 | Neuronal cargo accumulates at the tips of DA9 processes in wy743 mutants. (a) Schematic diagrams of the DA9 neuron. Inset shows the entire axon. (b) wt L4 larva (anterior is left and dorsal up in all figures) showing SVPs labelled by synaptogyrin/SNG-1::GFP. The cell body is marked by an asterisk. Lower panels show magnifications of the dendrite and the axon tip. Co-labelling with cytoplasmic mCherry (red) was used to identify the tip of the DA9 axon. Scale bar is $5 \mu \mathrm{m}$ in all panels and figures. (c) Schematic diagram of the ectopic SVP accumulations in wy743 dendrites. (d) synaptogirin/ SNG-1::GFP accumulates at the tips of wy 743 processes. White arrowhead indicates the dendritic accumulation (top and middle panel), and yellow arrowheads point to SNG-1::GFP that mis-accumulates at the tip of the axon (lower panel). (e) Intensity plot of synaptogyrin/SNG-1::GFP fluorescence in wt (grey) and wy743 (black) dendrites from $\mathbf{b}$ and $\mathbf{c}$, showing the enrichment of SVPs at the distal tip. (f) Dendrite length is reduced in wy 743 mutants. $n=30$ per genotype, $P<0.01, \chi^{2}$-test. ( $\left.\mathbf{g}, \mathbf{h}\right)$ The mitochondrial marker TOMM-20::YFP is evenly distributed in wt dendrites (indicated by arrows in $\mathbf{g}$ ) but accumulates at the tip in wy743 mutants (arrowhead in h). (i) In trak-1 mutants mitochondria do not enter the dendrite. (j) In wy 743; trak-1 double mutants, the mitochondria are confined to the cell body, suggesting that TOMM-20::YFP is correctly sorted. 
a

C. elegans wy743 VETAITTRLKSQLESSRNSNETESIFSRYN-ALFIRPRIRGAI

C. elegans VETAITTRLKSQLESSRNSNE⿵冂卄.

H. sapiens

M. musculus

D. rerio

D. melanogaster

S. cerevisiae
VETRITARIRDOLGTARNANE VETRI TARLRDQLGTARNANE UETRITARLRDQLGTAKNANEA E ESRFN-ALFVRPHIAGAI VETRI TAHLRDQL GTARNANE㓞 LEKKL QNTLASFNDSDHCPTEKL SYLVKFKPLMELCPRIKVKY $:{ }^{*}:{ }^{*} .::: \ldots{ }^{*}:::::{ }^{*}:{ }^{*}:$
PTRRKKMEAITTEUVHKR DTCCKLVSMR TRAANDFGWLOCMRE PIRRKKMEALITELVHKRDTCRKLVSMR IRAAND FGWLOCMRE PLRRRKLEHLITELVHQRDVTRSLIKSKIDNAKSFEWLSQMRE PLRRRKLEHLITELVHQRDVTRSLIKSKIDNAKS FEWLSQMRE GARRSKLNRI I IRF GHWSRVLNTPEKNMADHLRNIGMSYMSY PLRRRKLEHLINEFVHKRTVTRRLINNGVT SPRSFOWLCEMRE --- KKIEALLVEYLHFNNVIGQLKNCSTKEEARLIWAKVQKF

b wy743 (M563T) $\quad$ wy743 (R1782C) or352 (G2158D) or195 (S3200L) js319(S.A)

(N)

\begin{tabular}{|r|r|r|r|r|r|r|r|r|}
\hline Linker & AAA1 & 2 & 3 & 4 & Stalk & 5 & 6 & CT \\
\hline
\end{tabular}

C

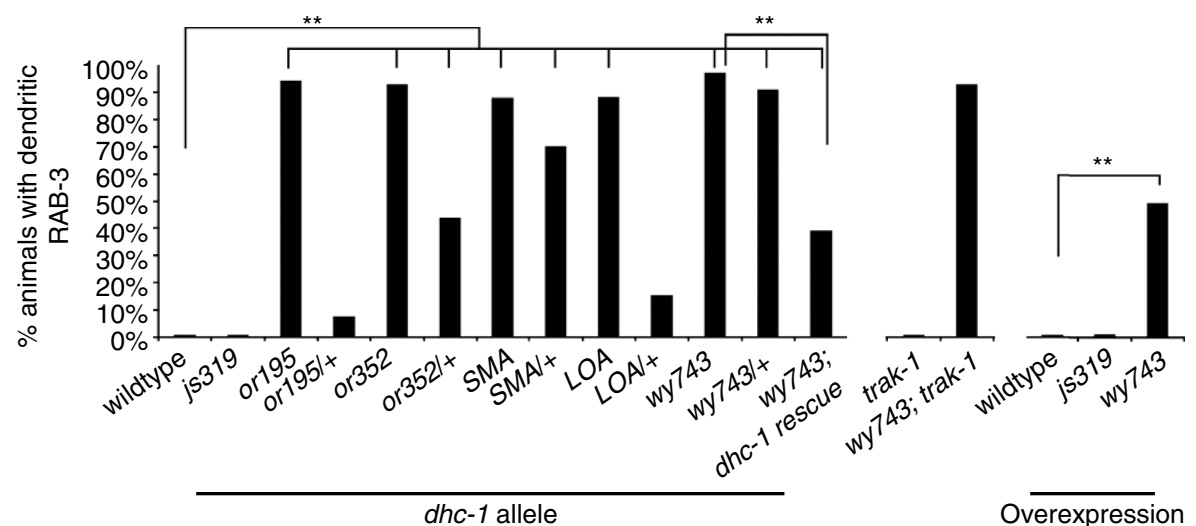

g
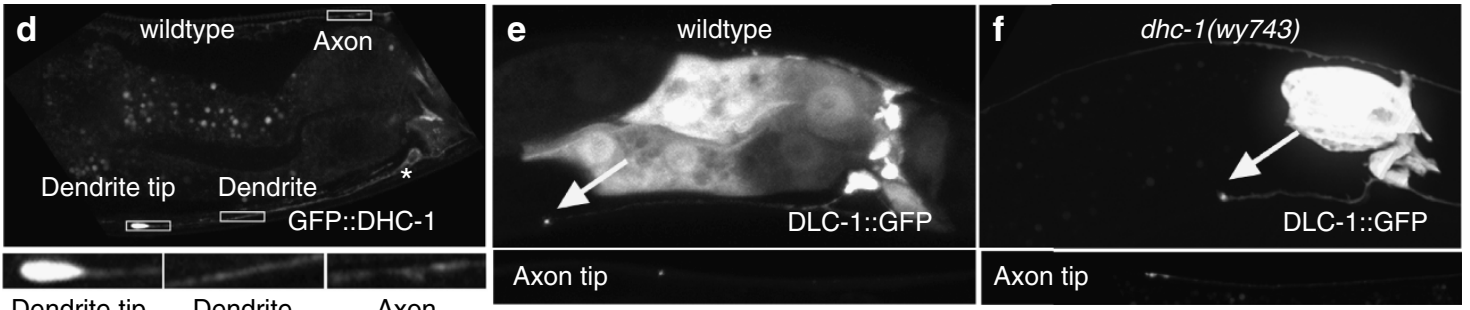

h

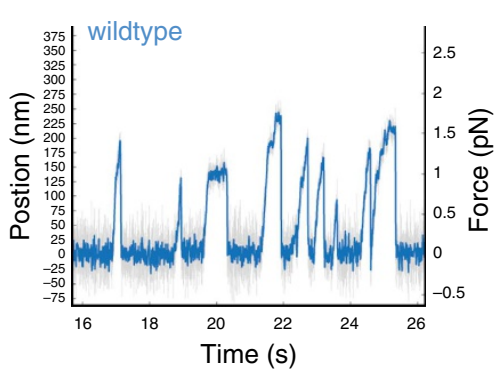

i

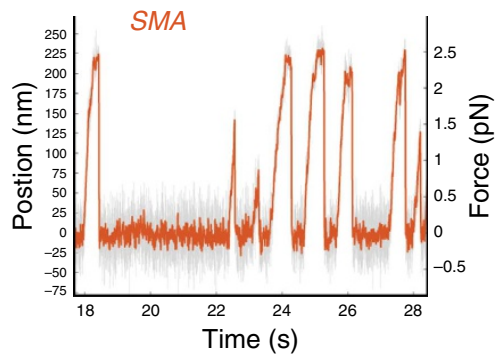

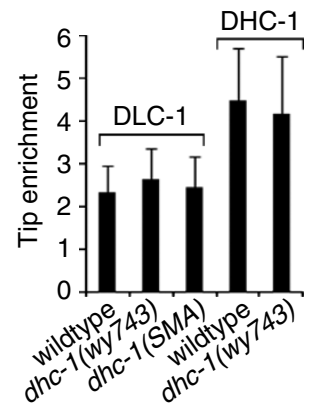

$d n^{-1} d n^{-1} d C^{-1}$

Dendrite tip Dendrite Axon

Axon tip

Axon tip

j

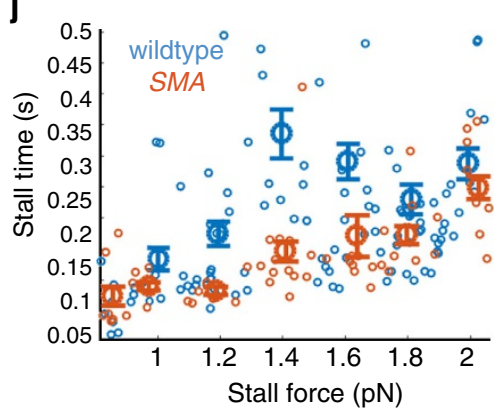

Figure 2 | wy743 is an allele of dhc-1. (a) Alignment of dynein heavy chain sequences encoding the $N$ terminal (left) and linker domains (right), shows their high conservation. The mutated residues in wy743 are highlighted. Boxes mark mutations that are associated with neuronal dysfunction in mice and humans. (b) Schematic drawing of DHC-1, with the conserved domains indicated. C. elegans mutations are indicated above, and the mouse and human mutations are marked beneath the scheme. (c) Quantification of dendritic SVP accumulations in dhc- 1 mutant alleles and transgenic rescue with genomic dhc-1 fragment. L4 and young adults were examined on a wide-field microscope, and any worm with a GFP::RAB-3 signal at the tip of the denrite was scored as positive. $n=27-60,{ }^{\star \star} P<0.01, \chi^{2}$-test. (d) Subcellular localization of a rescuing GFP::DHC-1 transgene expressed specifically in DA9. (e) DLC-1::GFP is also enriched at the dendrite tip and can be detected at the axon tip. (f) In dhc-1(wy743) mutants, the distribution of DLC-1::GFP is similar to wt. (g) Dynein tip enrichment was quantified as the ratio between GFP::DHC-1 or DLC-1::GFP in the tip and the entire dendrite, and normalized to a soluble mCherry marker. $n=17-23$. H,I. Representative stall-force traces for wildtype (h) and SMA (i) dynein immunoprecipitated with a FLAG tag from COS-7 cells. (j) Plot of stall-force vs. stall time showing that SMA dynein is less processive than wildtype $n=270$ (control) and 220 (SMA) from two independent experiments. $P<0.01$ for the range between 0.2 and $0.6 \mathrm{~s}$, ANOVA. Note that the range of forces observed is consistent with 1-2 motors. SA, splice acceptor. 
wy743 allele, but not wt or js319, resulted in dendritic accumulations that mimic the mutant phenotype (Fig. 2c). We conclude that $w y 743$ is a novel, dominant-negative allele of $d h c-1$.

We examined the subcellular localization of DHC-1 with a GFP::DHC-1 transgene expressed in DA9, and found that it is enriched at the dendrite terminal (Fig. 2d,g). We also monitored the localization of dynein light chain/DLC-1::GFP, a non-motor subunit, which depends on endogenous DHC-1's motor activity for its distribution, and is therefore a more reliable marker. As expected from a non-motor subunit, DLC-1::GFP showed more diffuse staining than GFP::DHC-1 but was still enriched at the dendrite tip in $85 \%$ of the animals (Fig. 2e,g). Axon tip enrichment was also frequently observed, consistent with reports from mammalian neurons ${ }^{29}$. These results indicate that a dynein pool exists at the dendrite tip, where defects arise in the mutant. DLC-1::GFP was not mislocalized in dhc-1(wy743) or dhc-1(SMA) mutants (Fig. 2f,g), suggesting that these alleles do not affect dynein's localization.

Cargo accumulation is not caused by transport defects. Accumulated cargo in the distal axon in $d h c-1$ mutants was attributed to transport defects, as axonal MTs are plus-end-out ${ }^{27}$. In contrast, as $90 \%$ of dendritic MTs in DA9 are minus-endout $^{54}$, accumulations in dendrites are inconsistent with impaired minus-end-directed motor activity.

We asked whether mitochondria mislocalization could underlie other defects in $d h c-1$ (wy743), but found that trak-1 mutants had no SVP accumulations, and trak-1 did not suppress the dhc-1(wy743) SVP accumulations (Fig. 2c) or MT phenotypes (Fig. 3c).

One possibility is that $d h c-1$ mutations cause MT polarity defects that lead to the dendritic phenotypes ${ }^{55}$. However, examination of MT polarity markers KLP-16::YFP and EBP-2:: GFP (ref. 54) did not reveal any polarity defects (Figs 3 and 6).

Another possibility is that wy743, LOA, SMA, or 195 and or352 are neomorphic alleles that bias DHC-1 dependent trafficking towards the MT minus-end. To investigate the relationship between transport defects and the accumulations, we imaged SVP trafficking in $d h c-1(w y 743)$ mutants and measured transport parameters on kymographs (Supplementary Fig. 3B). As a control we used wt animals and dhc-1(js319) mutants, in which trafficking is affected but no dendritic accumulations arise.

Retrograde speed in the axon, where MT are plus-end-out, was modestly decreased in $d h c-1$ (js319) mutants compared to wt, and it was further decreased in $d h c-1(w y 743)$ (Supplementary Fig. 3C). Since the MTs in the dendrites are minus-end distal, anterograde SVP movements are likely mediated by dynein. Consistently, anterograde speed was reduced in the dendrites of $d h c-1(w y 743)$ and $d h c-1(j s 319)$ compared to wt (Supplementary Fig. 3C). This indicates that changes in speed do not account for the accumulated dendritic cargo.

In the dendrite, we observed a similar small bias in directionality towards the minus end in both mutant alleles compared to wt (Supplementary Fig. 3E). Since $d h c-1(j s 319)$ has no accumulations, we conclude that changes in this parameter are also not responsible for the $d h c-1(w y 743)$ phenotype. Likewise, run length decreases were apparent in both mutants (Supplementary Fig. 3D). Together, these results indicate that transport towards the tip is reduced in both alleles. This decrease is inconsistent with the cargo buildup that occurs only in wy743. Hence, $w y 743$, and by inference from the steady-state phenotype, the LOA, SMA, or195 and or352 alleles, may also affect a transport-independent function of DHC-1.

MT defects correlate with accumulated cargo in $\mathbf{d h c}$-1 mutants. We next examined axonal MTs, labelled with GFP-tagged
Ensconsin MT Binding domain (EMTB::GFP) and GFP::TBA-1/ $\alpha$-Tubulin, and could not detect a difference between wt and dhc-1 mutants (Supplementary Fig. 4A-D). We then visualized dendritic MTs with the NCD-type minus-end-directed kinesin KLP-16::YFP, which is distributed on MTs in a distal to proximal gradient, causing it to be enriched at the dendrite tip ${ }^{54}$. In contrast to wt, dhc-1(wy743) mutants showed a prominent MT loop structure at the tip of the dendrite (Fig. 3a,b). The $d h c-1$ alleles SMA, LOA, or 195 and or352 showed a similar phenotype, which could be rescued cell autonomously (Fig. 3c). Additional MT markers (EMTB::GFP, GFP::TBA-1, and EBP-2::GFP) confirmed the presence of the loop at the dendrite terminal (Supplementary Fig. 4A-D).

We wondered whether MT looping and dendritic cargo accumulations could be related, since they arise at the same location. Consistently, the $d h c-1(j s 319)$ allele, which shows only axonal cargo accumulation, did not show dendritic MT abnormalities (Fig. 3c). Furthermore, examination of the MT loop in various $d h c-1$ heterozygous alleles revealed precisely the same spectrum of phenotypic penetrance as the SVP accumulation (Fig. 3c). These results indicate that MT defects in the dendrite correlate with cargo accumulation.

DHC-1 continuously maintains cystoskeletal integrity. Next, we studied the temporal progression of MT defects. A small loop at the tip of the dendrite was apparent in $15 \%$ of L2 larvae, $49 \%$ in L3 and all animals by L4. The appearance of dendritic SVP accumulations followed a similar developmental profile (Supplementary Fig. 4E). Along with dendrite growth, the size of the loop increased $(2.2 \pm 0.3 \mu \mathrm{m}$ in 1 day old adults, Fig. $3 \mathrm{~d})$. Adult animals occasionally had additional smaller secondary loops along the dendrite. However, as these loops were infrequent, we focused almost all our subsequent analysis on the terminal loop. With aging, the loop at the tip often collapsed and gave rise to other aberrant structures such as small branches or bifurcations (Fig. 3d). These observations suggest that $d h c-1$ is continuously required throughout dendrite growth. Consistently, cell-specific rescue of $w y 743$ was achieved with a transgene driven by the Pitr-1 promoter (Fig. 3c), which is only expressed after the mid L4 stage. Ongoing requirement for $d h c-1$ was also observed with the temperature-sensitive allele or195, which showed increased defects upon shift from $13{ }^{\circ} \mathrm{C}$ to $25^{\circ} \mathrm{C}$ at the $\mathrm{L} 4$ stage (Supplementary Fig. 4F). We conclude that $d h c-1$ is continuously required to maintain MT morphology and cargo distribution.

MT phenotypes correlate with reduced availability of SVPs. The progressive aggravation of the dendritic MT phenotypes in $d h c-1(w y 743)$ raises the possibility that cargo distribution defects are likewise exacerbated with age. We followed the accumulation of SVPs in the dendrite, in parallel with their loss from their normal location in the axonal synaptic patch. In wt animals, 90\% of synaptogyrin/SNG-1::GFP fluorescence was localized to the axon through the first 4 days of adulthood (Fig. 3e,f,h). In $d h c-1$ (wy 743$), 48 \%$ of synaptogyrin/SNG-1 fluorescence was localized to the dendrite in L4 animals. The accumulation of synaptogyrin/SNG-1::GFP in the dendrite increased over time, until at day $4,85 \%$ of the signal was in the dendrite (Fig. 3f,h). The accumulation of SVPs in the dendrite was accompanied by their disappearance from the synaptic patch (compare Fig. 3e,f). Hence, ectopic cargo accumulations in $d h c-1(w y 743)$ reduce the availability of SVPs at presynapses. $d h c-1(j s 319)$, which shows normal MTs but defective transport, had a phenotype similar to wt (Fig. 3g,h). Thus, maintenance of an intact dendritic cytoskeleton by DHC-1 is critical for ensuring normal levels of SVPs at presynapses. 

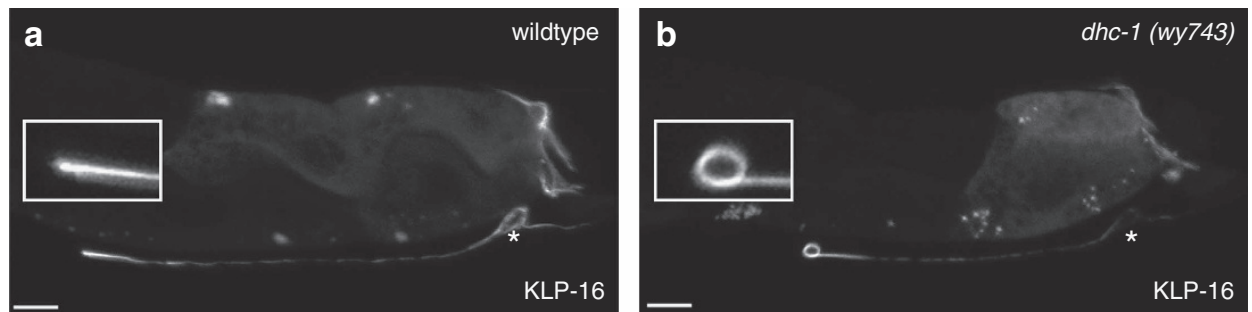

C
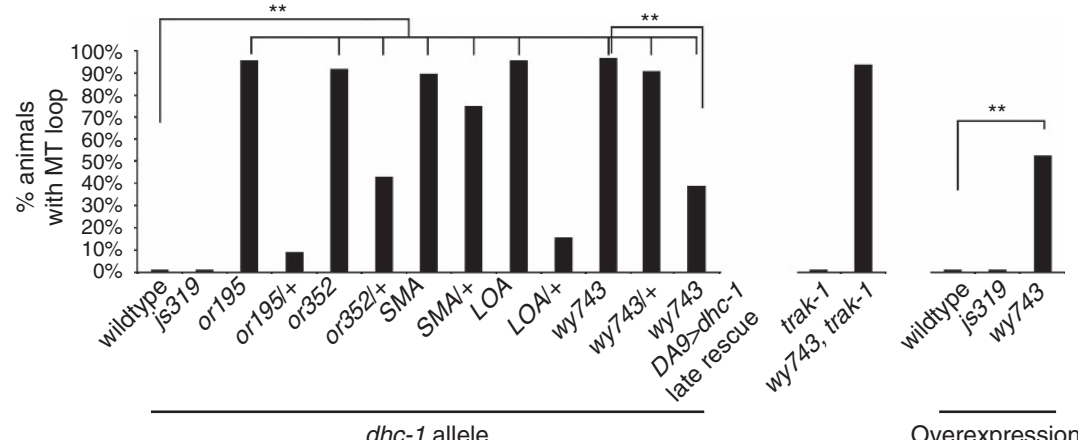

d
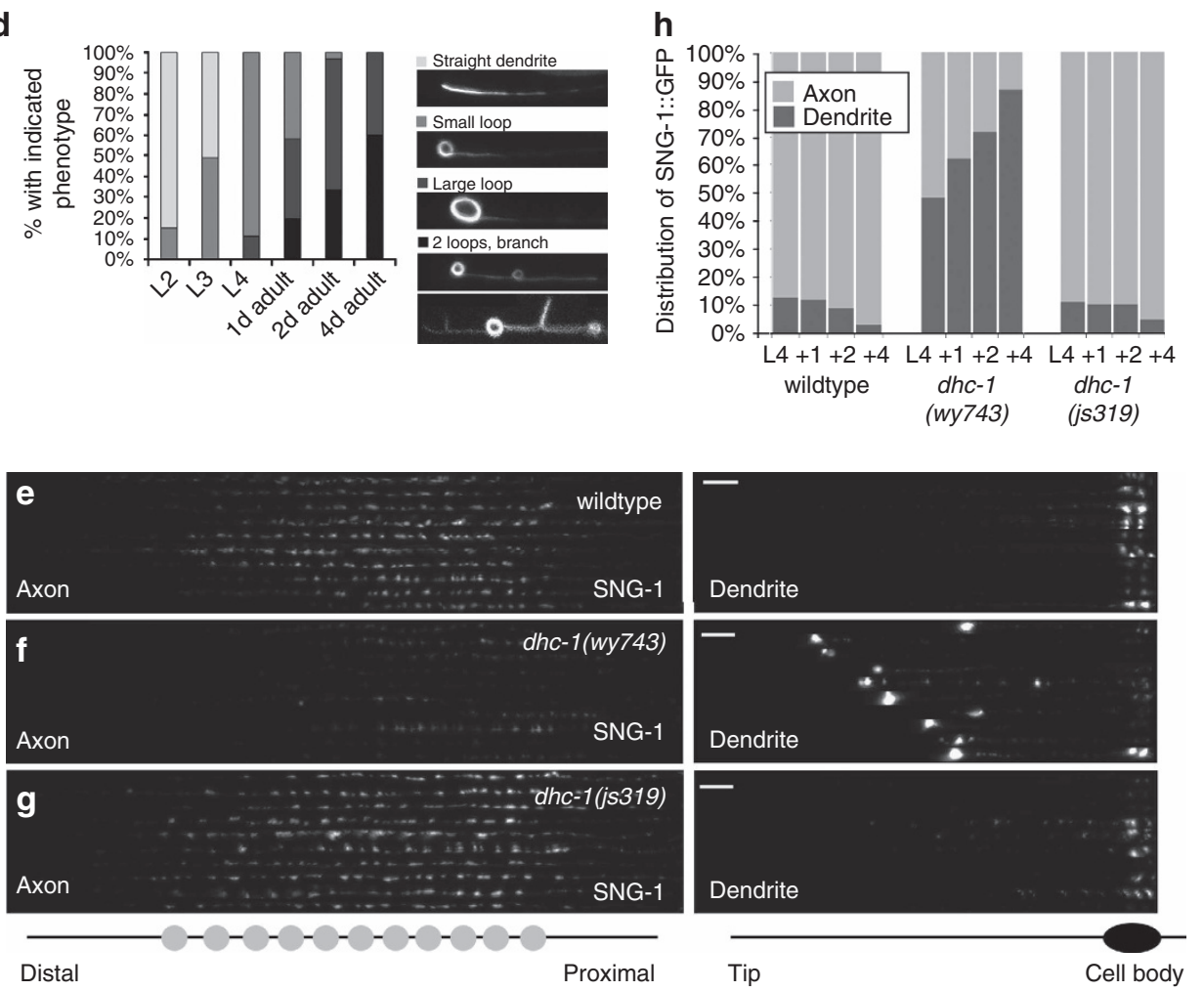

Figure 3 | Aberrant MT structures underlie SVP depletion from presynapses in dhc-1 mutants. (a,b) NCD/KLP-16::YFP fluorescence on dendritic MTs. KLP-16 is a minus-end-directed NCD-type motor, and is therefore enriched at the distal dendrite, reflecting the minus-end-out orientation of dendritic MTs. In a wt dendrite (a), MTs are straight. In dhc-1(wy743) (b), MTs show a prominent loop at the tip of the dendrite. (c) Quantification of dendritic MT loops in different $d h c-1$ alleles and DA9 specific rescue. $n=32-89$ per genotype, ${ }^{\star \star} P<0.01$. (d) Time course of MT morphological defects in dhc-1(wy743).

(e-g) Alignments of axons (left) and dendrites (right) taken from synaptogirin/SNG-1::GFP expressing worms at adult day 4 under identical settings. In dhc-1(wy743), the dendritic accumulations correlate with a depletion of SVPs from the presynapses. (h) Quantification of synaptogirin/SNG-1::GFP fluorescence in L4 through adult day 4 worms. dhc-1(wy743) gradually lose their synaptic SVPs as they accumulate in the dendrite. dhc-1(js319), similar to wt, maintains the dendrite/axon SNG-1::GFP ratio.

DHC-1 maintains the spatial distribution of MTs. Besides the soluble dynein fraction, a cortically anchored dynein pool can transport MT polymers ${ }^{41}$ or enhance synaptic stability ${ }^{38,41}$. To ask whether immobilized DHC-1 could be mediating the effect on MTs in DA9, we injected a construct harbouring a myristoylated, GFP-tagged version of DHC-1 driven by a DA9 promoter into dhc-1 mutants. Unlike GFP::DHC-1, which was cytoplasmic, the myritoylated construct showed a distribution 
that is consistent with membrane localization ${ }^{56}$ (Fig. 4a,b). Both constructs were expressed at comparable levels (Fig. 4c). We assayed the ability of both GFP::DHC-1 and myrGFP::DHC-1 to rescue axonal SVP accumulations, which are due to transport defect, in js319 and wy743 alleles, and observed no rescue with the myristoylated version (Fig. 4d). This suggests that while this construct may also generate a soluble DHC-1 pool that integrates into a dynein complex, it does not support efficient vesicular cargo transport. Despite this, the myrGFP::dhc-1 construct rescued loop formation in $d h c-1(S M A)$ and $d h c-1(w y 743)$ mutants (Fig. 4e). The rescue was incomplete, but comparable to the partial rescue obtained with non-myristoylated versions (Figs $2 \mathrm{c}$ and $3 \mathrm{c}$ ), which is likely due to the dominant nature of the alleles and their putative effect on dynein complex stability ${ }^{34,49}$. This result suggests that the dynein pool which prevents MT loop formation is not moving on MTs.

To better understand the effect of DHC-1 on MT organization in the dendrite, we measured average MT length, coverage (MT numbers at any point along the dendrite) and spacing (distance between minus ends) using a recently established method ${ }^{19,57}$. MT length, coverage and spacing along the entire dendrite were not dramatically affected in $d h c-1$ mutants (Supplementary Fig. 5). However, we noticed a significant distal shift in the distribution of MTs. We used the ratio between MT coverage at the distal dendrite (defined here as $5 \mu \mathrm{m}$, which is approximately the average MT length) and MT coverage at the rest of the dendrite to quantify this phenomenon. In wt, MT coverage at the distal dendrite was slightly lower than along the dendrite (Fig. 4f,g, Supplementary Fig. 5). In dhc-1 mutants, MT coverage is mildly reduced along the dendrite, and a concomitant increase of MT coverage is seen at the distal end (Fig. 4f-i, Supplementary Fig. 5). A similar trend was observed for heterozygous wy743 and SMA animals, in line with the dominant nature of these alleles. The skewed MT distribution suggests that $d h c-1$ is required to exert a force directed towards the cell body on the minus-end-out MTs in the dendrite. When $d h c-1$ function is
C
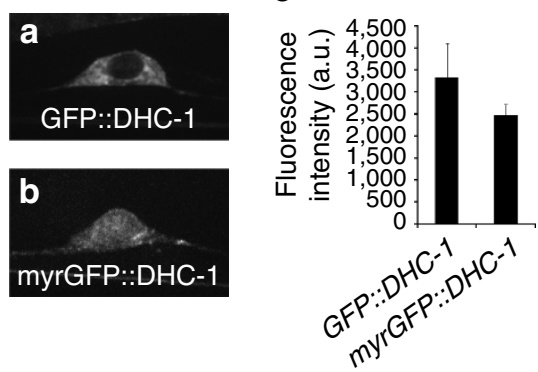

d

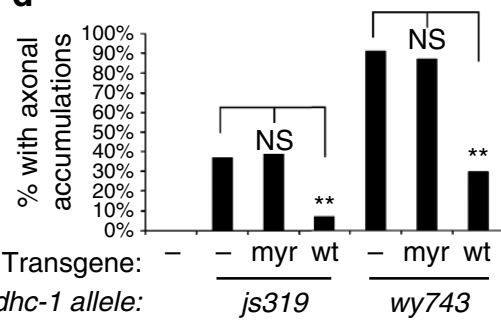

e

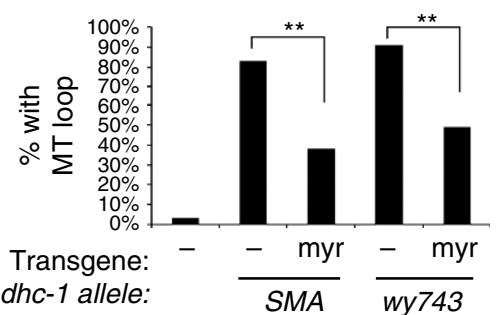

f

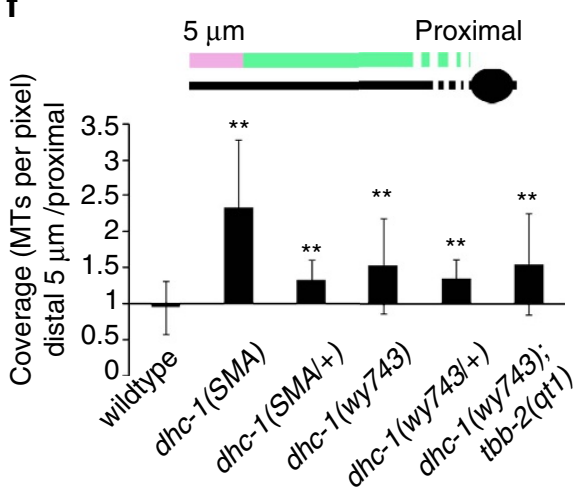

g

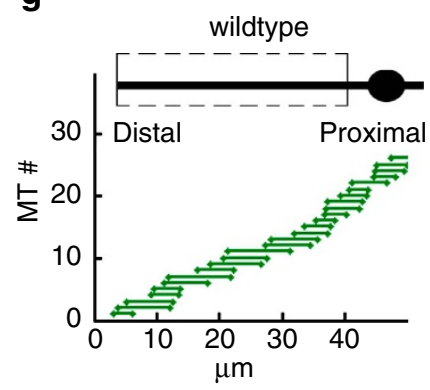

$\mathbf{h}$

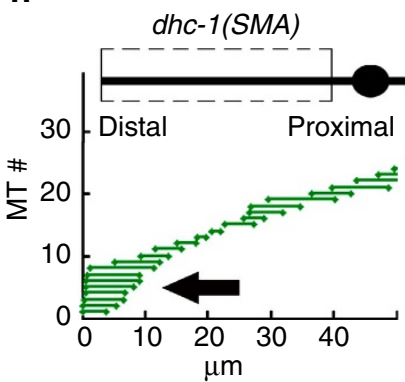

i

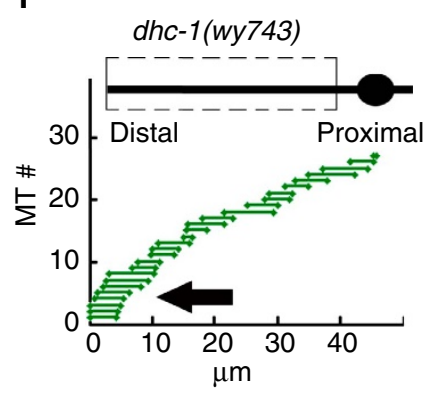

Figure 4 | dhc-1 cortical rescue and a distal shift in MT distribution in dhc-1 mutants. (a) GFP::DHC-1 is localized to the cytoplasm. (b) myrGFP::DHC-1 shows a distribution consistent with membrane localization. (c) Expression levels of GFP::DHC-1 and myrGFP::DHC-1 were quantified after imaging in identical settings. $n=17$ per genotype. (d) The myrGFP::DHC-1 construct does not rescue the accumulation of axonal SVPs (GFP::RAB-3). $n=20-25$, ${ }^{\star \star} P<0.05$. (e) membrane tethered myrGFP::DHC-1 rescues MT loop formation in dhc-1 mutants. $n=28-45$, ${ }^{\star \star} P<0.05$. (f) Ratio between MT coverage (number of MTs per pixel) in the distal $5 \mu \mathrm{m}$ of the dendrite to the rest of the dendrite. The results indicate that the distribution of MTs is shifted distally in dhc-1 mutants. (g-i) Representations of MTs in the dendrites of indicated phenotypes. The representation is derived from a quantized GFP::TBA signal, where steps up indicate a MT start and steps down a MT end. MT ends were randomly assigned to the longest MT in the bundle for representation purposes. See Methods for details. 
a
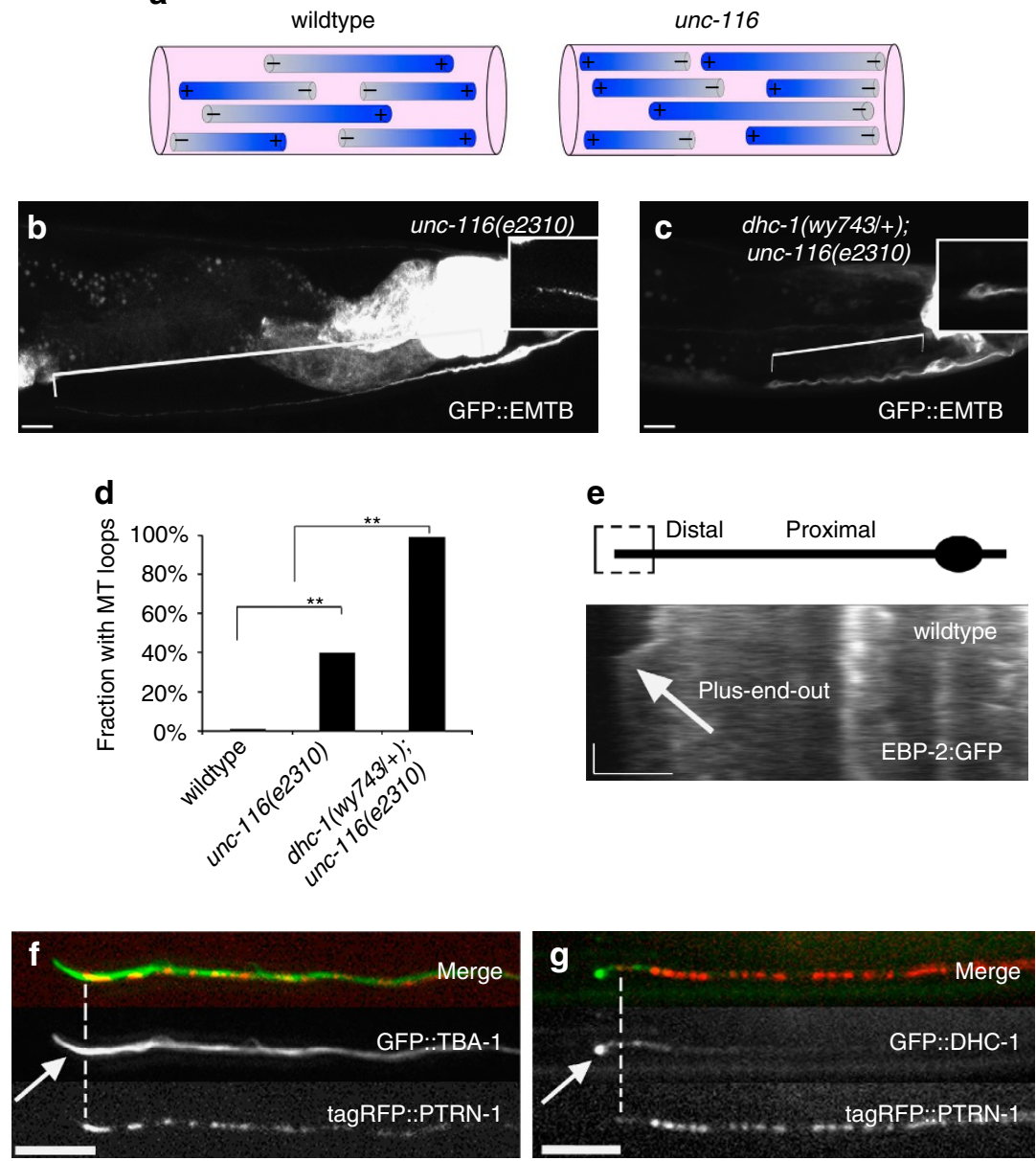

Figure 5 | DHC-1 acts on plus-end-out MTs. (a) Scheme illustrating MT polarity in wt dendrites (mixed, but mostly minus-end-out) versus unc-116(e2310) dendrites (uniformly plus-end-out). (b) MTs, labelled by GFP::EMTB, in unc-116(e2310) mutant dendrites. (c) In dhc-1(wy743/+); unc-116(e2310) double mutants the terminal MT loop is apparent in all dendrites despite the plus-end-out orientation of MTs. (d) Quantification of MT loops in the indicated genotypes. $n=25-31,{ }^{\star \star} P<0.01$. (e) A rare example of plus-end-out MT growth (labelled by EBP-2::GFP) at the tip of a wildtype dendrite. (f) tagRFP::PTRN-1 labels MT minus-ends. The presence of the MT marker GFP::TBA-1 after the distal-most tagRFP::PTRN-1 punctum indicates the presence of plus-end-out MTs at the dendrite tip. (g) GFP::DHC-1 accumulates distal to the last tagRFP::PTRN-1 punctum, suggesting that dynein is positioned in a location that allows it to interact with MT plus-ends.

compromised, the distribution of dendritic MTs is skewed distally, possibly by actin based forces ${ }^{35}$ or a MT motor ${ }^{38}$.

DHC-1 acts on plus-end-out MTs to prevent loop formation. The distal enrichment of MTs in $d h c-1$ mutants may lead to looping. This model predicts that looping would depend on MT polarity, as in the dendrite dynein would push minus-end-out MTs towards the cell body and plus-end-out MTs outwards. We therefore examined unc-116(e2310)/kinesin-1 mutants, in which dendritic MT polarity is flipped to a plus-end-out configuration $^{54}$. In this background, an anchored DHC-1 is expected to push MTs distally, and MTs should accumulate proximally in a dhc-1 mutant. dhc-1(wy743); unc-116(e2310) double mutants were lethal, so we used $d h c-1(w y 743 /+)$; unc-116(e2310), as $d h c-1(w y 743 /+)$ heterozygotes still show the MT loop. The double mutant maintained the unc-116/kinesin-1 phenotype of reversed MT polarity (Supplementary Fig. 6A-C). Surprisingly, we found that in $u n c-116$ mutants, $d h c-1$ was still required to prevent MT loop formation (Fig. 5a-d). This suggests that preventing MT looping by $d h c-1$ does not require minus-end-out MTs. Since only minus-end-out MTs are expected to shift distally in $d h c-1$ mutants, this result also suggests that the loops are not caused by the distal MT shift. Likewise, this reinforces the notion that transport defects do not account for the MT loops, as DHC-1 is expected to transport cargo towards the cell body in unc-116(e2310). Interestingly, some MT loops that accumulated cargo were observed in unc-116 single mutants (see Fig. $5 \mathrm{~d}$ for MT loops). This effect is likely due to reduced levels of dynein at the dendrite tip in these mutants, as it does not move anterogradely on a plus-end-out array ${ }^{54}$, and would not be efficiently transported by mutant unc-116/kinesin-1 (refs 58,59). Consistently, DLC-1::GFP was reduced, but not eliminated at the dendrite tip in unc-116 mutants (Supplementary Fig. 6D-F).

Since these results suggest that plus-end-out MTs are mediating loop formation in $d h c-1$ mutants, we asked whether such MTs are found at the dendrite tip. We examined MT polarity using GFP::EBP-2, which binds to the growing ends of $\mathrm{MTs}^{60}$. MTs at the dendrite tip were extremely static and rarely showed dynamic behaviour that would indicate their polarity. However, we could observe rare growth events, which were directed outwards (Fig. 5e). Plus-end-out growth at the dendrite tip was also observed in efa-6 mutants (Supplementary Fig. 7C), which were reported to increase MT dynamics ${ }^{61}$.

We next examined MTs that were marked with GFP::TBA-1 and decorated with RFP::PTRN-1, which binds minus ends ${ }^{62-64}$. 
GFP::TBA-1 often extended beyond the distal-most RFP::PTRN-1 punctum, indicating the presence of plus-end-out MTs at the dendrite tip. Similarly, GFP::DHC-1, which accumulates at the tip of the dendrite, was often found beyond the distal-most RFP::PTRN-1 punctum (Fig. 5f,g).

Since plus-end-out MTs are sufficient for loop formation in dhc-1(wy743/+); unc-116(e2310) mutants, we wondered if the loops could also form at axonal tips, where MTs are oriented plus-end-out. No loops were detected in $d h c-1$ mutants at the tip of the DA9 axon (Supplementary Fig. 4). However, in DA1, the anterior-most DA neuron, MT loops were present in dhc-1(wy743) mutants but not in wt (Supplementary Fig. 7A,B). This result confirms that plus-end-out MTs mediate the $d h c-1$ phenotype.

DHC-1 suppresses MT dynamics at the distal dendrite. To understand the effect of DHC-1 on MT dynamics, we monitored the + TIP protein EBP-2::GFP (Supplementary Movies 1 and 2) and analysed the resulting kymographs ${ }^{60}$. As a second readout we used a GFP::TBA-1, which labels MTs uniformly. RFP::TBA-1 and EBP-2::GFP colocalize during MT growth ${ }^{19}$. Consistent with previous studies ${ }^{19,54}$, DA9 MTs showed a low frequency of EBP-2::GFP comets. We divided the analysis into three sections: the dendrite tip, the distal $5 \mu \mathrm{m}$ and the proximal part extending to the cell body (Fig. 6a). In wt, MTs grew primarily at the proximal region. Fewer EBP-2::GFP comets were observed in the distal $5 \mu \mathrm{m}$, and almost none at the tip (Fig. 6b,g, Supplementary Movie 3). dhc-1 mutants had normal MT dynamics near the cell body, mildly elevated dynamics at the distal $5 \mu \mathrm{m}$ and a drastic increase at the tip (Fig. 6c,d,g, Supplementary Movies 4 and 5). Particularly striking was the increase in plus-end-out EBP-2::GFP comets at the tip (Fig. 6d), which contrasts with almost none in wt. Elevated MT coverage may explain the increase in EBP-2::GFP comets at the distal $5 \mu \mathrm{m}$ but not at the tip, where the change is much higher than the $\sim$ twofold increase in polymer numbers. Furthermore, plus-end-out MTs are predicted to be pushed towards the cell body in $d h c-1$ mutants, suggesting that the increase in plus-end-out MT growth at the tip is not related to the skewed MT distribution.

Neuronal stress may increase MT dynamics through JNK signalling ${ }^{65}$. However, increased MT dynamics, looping and cargo accumulation were all maintained in dhc-1(SMA); jnk-1(gk7) double mutants (Supplementary Movie 8). These observations suggest that DHC- 1 maintains MTs in a static state, and that this function is particularly relevant for plus-end-out MTs at the dendrite tip.

Observations with GFP::TBA-1 and EMTB::GFP revealed that excessive growth in dhc-1 was accompanied by excessive shrinkage, resulting in repeated cycles of loop formation and collapse (Fig. 6f,h, Supplementary Movie 6).The speed and extent of MT growth and shrinkage were not altered in $d h c-1$ mutants (Supplementary Fig. 8). In both wt and mutant, growth events were coupled to shrinkage, such that overall polymer content did not change significantly (Supplementary Fig. 8), consistent with the quantification of MT coverage (Supplementary Fig. 5). These results suggest that $d h c-1$ is required to inhibit dynamic behaviour, not to prevent MT growth per se.

Loop formation in $d h c-1$ mutants involved MT buckling and then twisting (Fig. 6h, Supplementary Fig. 9A, Supplementary Movies 6 and 7), which may result from excessive forces that push the MTs distally. Alternatively, plus-end-out MTs growing against a barrier could buckle and twist to form a loop when dynein is not found on the barrier to tether them ${ }^{43,44,66}$. To distinguish these possibilities we bleached a small region proximal to the loop, such that under the first model, the marked MTs should be observed moving into the loop as it collapses and reforms (Supplementary Fig. 9B). Despite abundant MT dynamics in the loop, we did not observe the marked MTs moving into the loop (Supplementary Fig. 9C). This indicates that loop formation and collapse do not involve MTs moving distally, consistent with a mechanism that acts locally.

Dynamic MTs cause structural defects and cargo accumulation. MT instability, loops and cargo accumulation in dhc-1 mutants may arise independently, or be causally related. We noticed that in 3-day-old adult $d h c-1$ (wy743) worms secondary loops accumulated SVPs, suggesting that MT abnormalities and cargo accumulation are related (Fig. $7 \mathrm{a}$ ).

To test whether MT instability in dhc-1(wy743) leads to MT loops, we grew mutant animals at increasing concentrations of Taxol. Taxol efficiently suppressed EBP-2::GFP comets in wt or dhc-1(wy743) mutants (Fig. 7c). Concentrations up to $1 \mu \mathrm{M}$ only rescued the loop in a small fraction of animals (15\% rescue at $1 \mu \mathrm{M})$, whereas higher concentration were lethal to $d h c-1(w y 743)$ homozygotes $(95 \%$ lethality at $2 \mu \mathrm{M})$. We therefore examined heterozygotes, which still show the mutant phenotype in almost all animals. At $2 \mu \mathrm{M}$ taxol in the culture medium, $45 \%$ of the animals showed a straight dendrite, suggesting that excessive MT dynamics causes the loops (Fig. 7b,d).

We next examined a temperature-sensitive allele of $\beta$-tubulin, tbb-2(qt1), in which an E198K substitution increases MT stability in C. elegans and Aspergillus nidulans ${ }^{67,68}$. The double mutant dhc-1(wy743); tbb-2(qt1) had significantly reduced MT dynamics compared to dhc-1(wy743) single mutants, indicating that tbb-2(qt1) can also stabilize neuronal MTs in $d h c-1$ mutants (Fig. 7c,e,f).

The percentage of $d h c-1(w y 743)$; tbb-2(qt1) double mutants that showed the terminal loop was significantly reduced compared to $d h c-1$ (wy743) single mutants (Fig. 7d), strengthening the hypothesis that unstable MTs cause the abnormal dendritic morphology. tbb-2(qt1) did not suppress the distal skew of MT density in $d h c-1$ mutants (Fig. 4f), indicating that the role of DHC- 1 in pushing MTs towards the cell body can be genetically uncoupled from its role in suppressing MT dynamics.

Lastly, we tested whether stabilizing MTs could also rescue the ectopic accumulation of SVPs in dhc-1 mutants, and found that to be the case (Fig. $7 \mathrm{~g}-\mathrm{j})$ : the fraction of $d h c-1(w y 743)$; tbb-2(qt1) animals with accumulations was reduced by $40 \%$, and in the animals that did show accumulations, the intensity of the GFP::RAB-3 at the dendrite was diminished compared to dhc-1(wy743) single mutants. Similarly, dhc-1(wy743); tbb-2(qt1) double mutants showed weaker accumulations of mitochondria at the tip of the dendrite compared to dhc-1(wy743) single mutants. This effect was accompanied by appearance of mitochondria at their normal location along the dendrite (Supplementary Fig. 10).

Together, these results suggest that DHC-1 prevents MTs at the dendrite tip from becoming excessively dynamic, and that MT structure and cargo distribution are aberrant when this stability is compromised.

\section{Discussion}

Dynamic MT arrays are important during cell division, while stable MTs are required to support the long-range trafficking characteristic of neurons. Our results illustrate the consequences of a loss of MT stability in the dendrite. In dhc-1 mutants, MTs become excessively dynamic, resulting in aberrant morphology and trapping of cargo at the dendrite tip at the expense of normal locations. Two trafficking-independent functions of DHC-1 shape the dendritic cytoskeleton: control of MT distribution and 
suppression of MT dynamics at the dendrite tip. We propose a model where the latter function is carried out by an interaction between anchored dynein and MT plus-ends.
Dynein's best described function is retrograde transport ${ }^{25-29}$. Although cargo mislocalizes in $d h c-1$ alleles, several observations suggest that transport defects do not drive this phenotype:

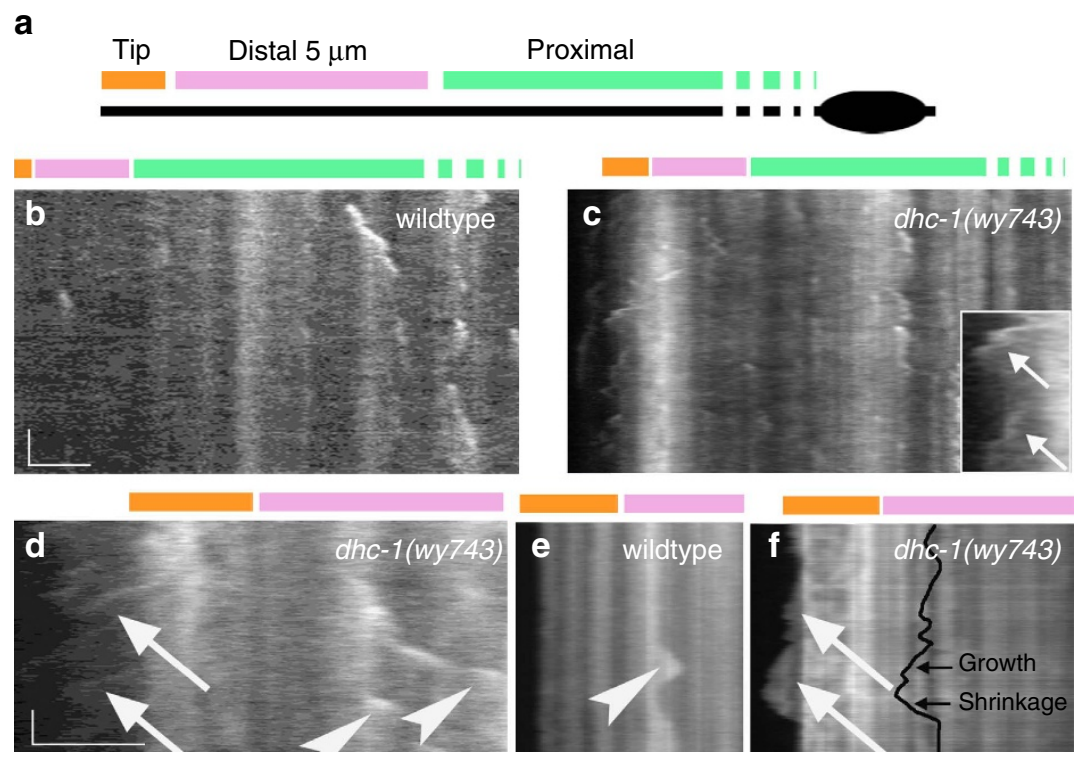

Plus-end-out

Minus-end-out

g

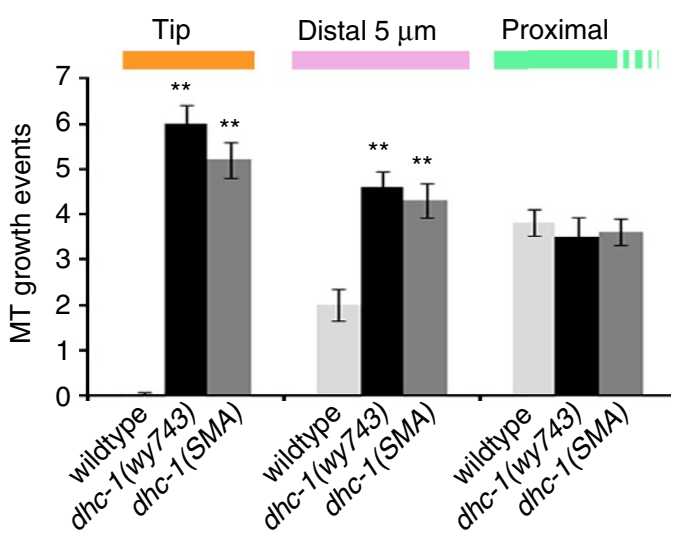

h

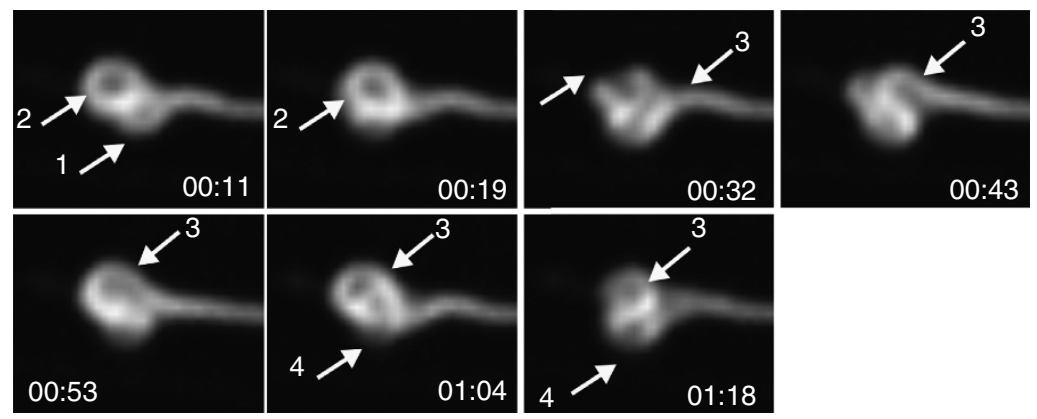

Figure 6 | Highly dynamic plus-end-out MTs at the tip of the dendrite in dhc-1mutants. (a) Scheme illustrating the division of the dendrite into three areas. (b) Kymograph from EBP-2::GFP movies of a wt L4 animal. MT growth is detected mostly near the cell body. (c) MT dynamics are increased in the distal dendrites of dhc-1 mutants, particularly at the tip, where abundant plus-end-distal growth is observed (arrows). (d) An enlarged view of the distal dendrite from a dhc-1(wy743) animal. Abundant plus-end-out growth is detected (arrows). Before the tip, an elevation in the number of plus-end-proximal EBP-2::GFP comets (arrowheads) mirrors the increase in MT coverage of this area. (e) GFP::TBA-1 movie of wt reveals that growth of minus-end-out MTs (arrowhead) at the distal dendrite is coupled to shrinkage. No growth is observed at the tip. (f) Plus-end-distal growth of MTs (arrow) in the terminal loop of dhc-1 mutants, visualized with GFP::TBA-1, is coupled to shrinkage. (g) The number of MT growth events (defined as either an EBP-2::GFP comet or a GFP::TBA-1 growth signal) in dhc-1 mutants is mildly elevated in the distal dendrite and drastically elevated at the tip. $n=24-37$ movies per genotype. ${ }^{\star \star} P<0.01$. See Supplementary Fig. 8 for quantifications of additional dynamic parameters. (h) Single frames from a GFP::TBA movie of dhc-1(wy743) showing the formation and collapse of four MT loops (arrows). The buckling of loop 3 and the collapse of loop 2 are particularly evident. 

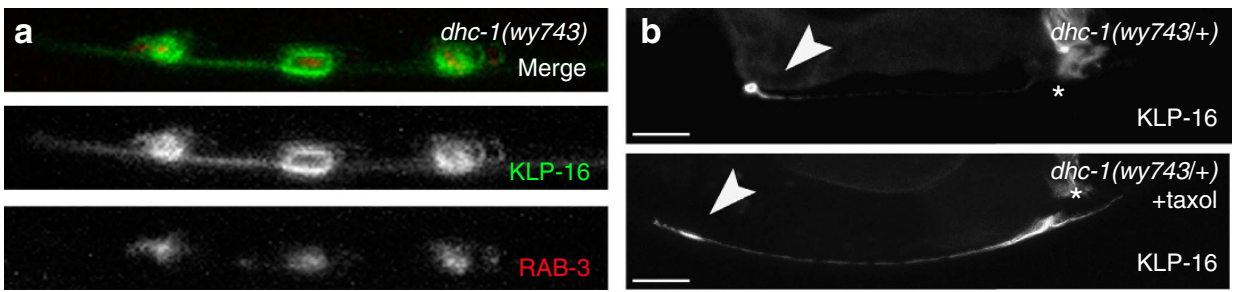

C

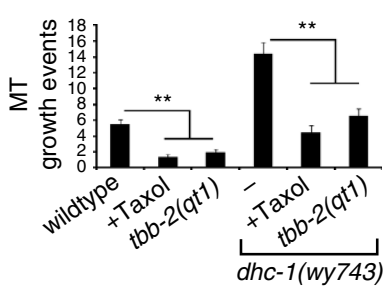

d

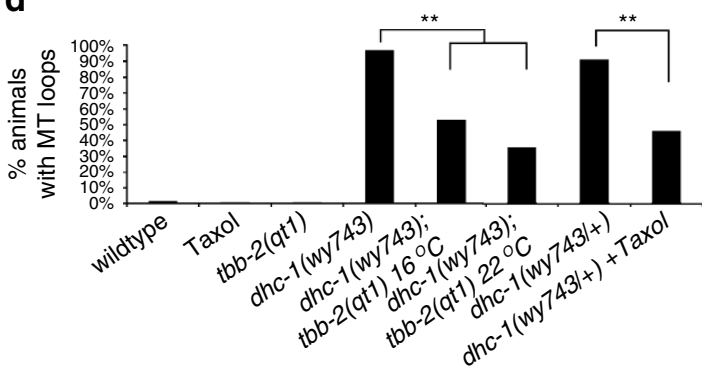

$\mathbf{f}$
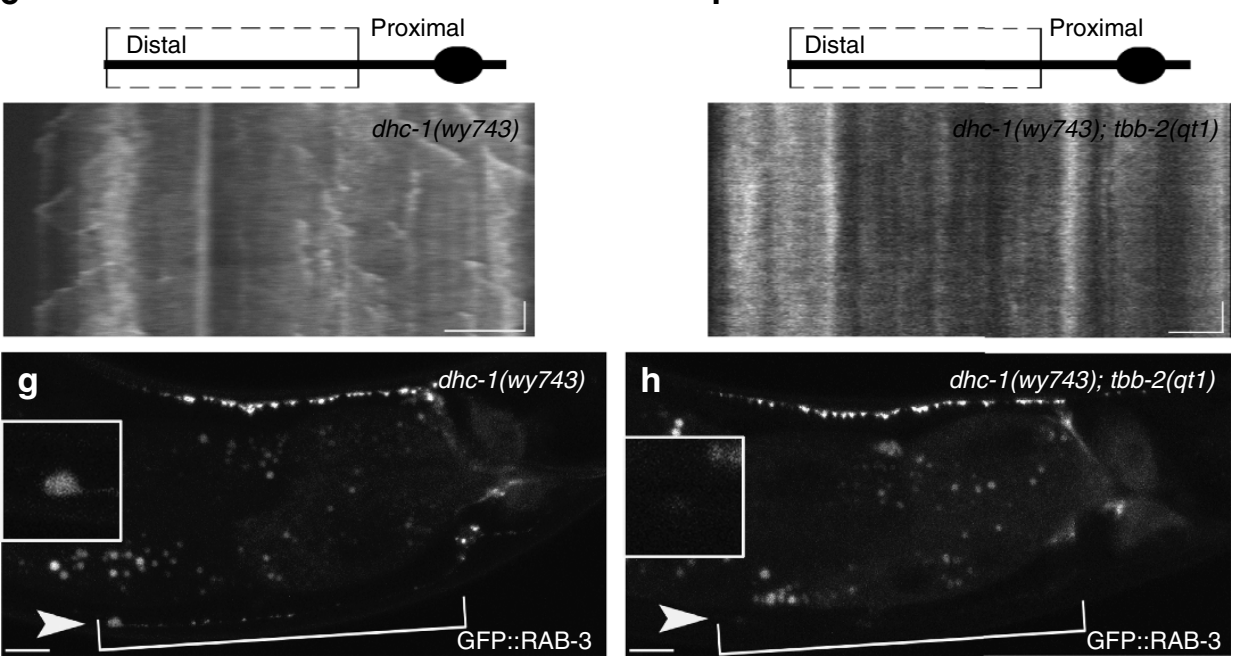

i

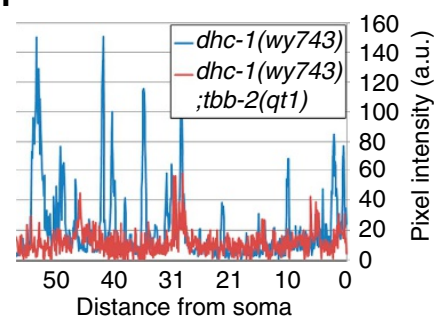

j
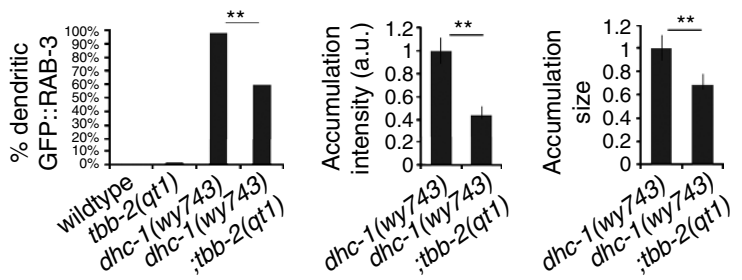

Figure 7 | MT instability is the cause of structural defects and cargo accumulation in dhc-1 mutants. (a) SVPs, marked by RAB-3::TdTomato (red) colocalize with secondary MT loops (NCD/KLP-16::YFP, green) in the dendrite of 3-day-old dhc-1(wy743) mutants. (b) MT stabilization by Taxol rescues loop formation in $d h c-1($ wy $743 /+)$ ) animals. (c) Quantification of MT dynamics in the presence of Taxol or the MT stabilizing mutation tbb-2(qt1) suggests that $t b b$-2(qt1) effectively stabilizes neuronal MTs in DA9. $n=20-26$ kymographs PER genotype, ${ }^{\star \star} P<0.01$. (d) Quantification of the suppression of the terminal MT loop in dhc-1 mutants by Taxol, or with the MT stabilizing mutation tbb-2(qt1).Note that some suppression occurs at the permissive temperature. This may reflect either a partial loss of function at the permissive temperature, or the fast-acting nature of this allele ${ }^{52} . n=31-39$ animals per genotype, ${ }^{\star \star} P<0.01$. (e,f) Kymographs showing the stabilizing effect of tbb-2(qt1) on MTs in dhc-1 mutants. dhc-1(wy743) mutants (e) show abundant MT growth, at the loop and the distal dendrite. This growth is suppressed in the double mutant dhc-1(wy743); tbb-2(qt1) (f). (g,h) Suppressing MT dynamics in dhc- 1 mutants abrogates cargo accumulation at the tip of the dendrite. dhc-1(wy743) mutants ( $\mathbf{g}$ ) show the typical SVP accumulation (markerd by GFP::RAB-3) at the tip of the dendrite. In dhc-1(wy743); tbb-2(qt1) double mutants (h) the accumulations are strongly reduced. (i) Fluorescence intensity profile of GFP::RAB-3 in the dendrite shows that SVP accumulations in dhc-1(wy743) are effectively eliminated by tbb-2(qt1) induced MT stabilization. (j) Quantifications of the frequency, size (normalized) and intensity of GFP::RAB-3 accumulations. The MT stabilizing mutation tbb-2(qt1) effectively reduces all these parameters in dhc-1(wy743) mutants. $n=23-25$ images per genotype, ${ }^{\star \star} P<0.01$. 
(1) dendritic MTs are minus-end-out; therefore, reducing minus-end-directed transport should lead to accumulations in the cell body, not the tip. (2) The js319 allele is defective for SVP transport yet shows no tip accumulations. (3) Cargo accumulation can be rescued by MT stabilization. A related hypothesis, that dynein transports an unknown MT stabilizer to the dendrite tip is also inconsistent: (1) in this scenario, the js319 allele would show MT loops. (2) Flipping MT polarity in unc-116/kinesin-1 mutants would eliminate the requirement for $d h c-1$ in preventing MT loops. (3) The phenotype can be rescued by a myrisotylated DHC-1. In axons, forces exerted by anchored dynein push MTs towards their plus ends, and are opposed by myosin or kinesin dependent forces ${ }^{35-38}$. Our results suggest that a similar mechanism operates in the dendrite, accounting for the skewed MT density and the $\sim$ twofold increase in MT dynamics at the distal $5 \mu \mathrm{m}$ of $d h c-1$ mutants. However, MT movement cannot explain the loop or cargo accumulation: it is inconsistent with the suppression of the loop by taxol. Furthermore, the fact that $t b b-2$ (qt1) rescues loop formation and cargo accumulation but not the shifted MT distribution indicates that the latter defect does not account for former.

The rescue experiments with taxol and $t b b-2(q t 1)$ indicate that MT stabilization at the dendrite tip is required to prevent loop formation and cargo accumulation. Mechanistically, we favour a model where stabilization is carried out by immobile DHC-1 tethering the MT plus-ends at the dendrite tip. This is consistent with requirement for $d h c-1$ on a plus-end-out MT array (Fig. 5, Supplementary Fig. 7), with the presence of non-dynamic plus-end-out MTs at the dendrite tip in wt (Fig. 5) and with the local increase in plus-end-out MT dynamics in $d h c-1$ mutants (Fig. 4). Furthermore, this model is consistent with the accumulation of cargo at the dendrite tip despite reduced transport towards this location (Fig. 2, Supplementary Fig. 3), and with the rescue by myrGFP::DHC-1 (Fig. 4). Previous observations of tethering suggest that dynein interacts with MT plus-ends transiently to stabilize their dynamics ${ }^{41,43,44}$. Reduced processivity may affect tethering by reducing the time that dynein remains engaged with the plus end of the MT. Although dynein's localization suggests that this function is primarily carried by a pool at the tip of the dendrite, our data cannot exclude similar DHC-1 activity throughout the dendrite. In fact, this interpretation is consistent with the observed shift in MT density and with the low penetrance phenotype of secondary loops. Nevertheless, the appearance of all the related phenotypes at the dendrite tip suggests that the requirement for $d h c-1$ dependent MT tethering and stabilization is most significant at this location.

The link between MT abnormalities, axonal transport and neuronal disease is becoming increasingly clear ${ }^{30,31}$. The dhc-1(SMA) allele suggests a possible cellular mechanism for disease-linked DYNC1H1 mutations: in the absence of MT tethering/stabilization, excessively dynamic MTs may impair the distribution of neuronal cargo.

\footnotetext{
Methods

Imaging. Worms were paralyzed in $3 \mathrm{mM}$ levamisole and mounted on $2 \%$ agar pads. For time lapse movies, worms were paralyzed in $0.4 \mathrm{mM}$ levamisole for $15 \mathrm{~min}$, then mounted in M9 buffer on $10 \%$ agarose pads.

Imaging was done on a Zeiss LSM710 scanning confocal microscope using a Plan-Apochromat $40 \times 1.3$ objective. For time lapse movies, a Zeiss Axio Observer equipped with a Plan-Apochromat $63 \times 1.4$ objective, a Yokogawa spinning disc and $488 \mathrm{~nm}$ laser line was used. The Mosaic system (Andor) was used for photobleaching with a $405 \mathrm{~nm}$ laser line. For SVP trafficking movies, we used streaming mode with $100 \mathrm{~ms}$ exposure. For MT dynamics, we used a frame rate of $7 \mathrm{~s}^{-1}$. Movies were $40 \mathrm{~s}$ to $1 \mathrm{~min} 30 \mathrm{~s}$ long depending on the experiment.
}

Image analysis. For dynamic imaging, analysis was carried out using ImageJ. Images were first corrected for animal movement using the ImageJ plugin StackReg. The axon/dendrite was then traced and Kymographs were generated using the 'reslice' function. For analysing MT dynamics at the tip of the dendrite, where the looping occurs, we only used animals where we could confidently follow the MTs (that is, determine proximal versus distal) throughout the loop.

Single events were traced manually on the kymographs and speed, run-length and directionality were measured using the 'measure' function. We measured all visible events that were longer than $200 \mathrm{~ms}$. Data was normalized by time and size of the imaged neurite segment. Quantifications of puncta size and intensity were carried out on images acquired under identical settings using the Analyze/Measure function in imageJ. Statistical tests and graphs were generated with Excel. Images were assembled into multi-panel figures using Photoshop.

Analysis of MT organization: this method is described in a separate

manuscript ${ }^{19}$. Only a brief description is provided here. More detailed information is available upon request.

Worms that express GFP::TBA-1 and tagRFP::PTRN-1 specifically in DA9 were generated. The integration of GFP::TBA-1 into the MTs was measured by FRAP. tagRFP::PTRN-1 labels discrete puncta that correspond to MT minus-ends. A custom Matlab code then analyzes the images.

Briefly, the analysis consists of the following steps: (1) background subtraction. (2) A user dawn line is used to generate a mask of the neuronal process. The algorithm adjusts the mask using a reiterative process and sums all pixels intensities at each location along the mask. (3) Single MT fluorescence intensity extraction. In this step the code scans a range of possible intensities, and each one is used to quantize the GFP line scan. The intensity that minimizes the difference between the number of steps up on the quantized distribution and the number of observed tagRFP::PTRN-1 puncta is selected as the intensity of a single MT and is used to calculate the MT organization parameters. (4) Calculation of MT organization parameters: spacing of minus ends is calculated as the average distance between tagRFP::PTRN-1puncta in the line scan. Coverage is calculated by summing the GFP intensity along the process and dividing it by the previously calculated intensity of a single MT. The result is divided by the length of the scan to obtain the average MT numbers per pixel. Average MT length is obtained by dividing the total coverage by the number of MTs in the line scan.

All images analysed were manually verified and we discarded images with worm movement, a too dim signal or an inconsistent signal due to variations in distance of the process from the objective.

The method was validated by several means: (1) the results for the single MT intensity were similar to measurement of this parameter on kymographs, where the growth of a single MT allows to determine its fluorescence intensity. (2) Results for average MT length and coverage were in agreement with previously published EM reconstructions. (3) We performed an EM reconstruction of $10 \mu \mathrm{m}$ in DA9 in one animal, as well as correlative fluorescence/EM analysis of $7.5 \mu \mathrm{m}$ in another animal. In both cases the results of the fluorescent method were in good agreement with the EM reconstruction.

Genetic screen and generation of dhc-1(SMA) dhc-1(LOA) alleles. All worm strains are listed in Supplementary Table 1. The wy743 allele was isolated from a semiclonal F2 EMS screen of 2000 haploid genomes. SNIP-SNP mapping and rescue was performed using standard protocols. Whole genome sequencing was performed on Illumina $\mathrm{Hi}$-seq platform and sequence analysis was carried out using CLC Genomics Workbench 5.

The $d h c-1(S M A)$ and $d h c-1(L O A)$ allele were generated by a CRISPR-Cas9based methodology. We used two sgRNAs closely flanking the mutation, and a $200 \mathrm{bp}$ oligo(LOA) or a PCR product (SMA) as a repair template. The presence of the mutation was verified by sequencing and the mutants were outcrossed four times to eliminate off-target mutations.

Dynein force measurements. COS-7 cells were transfected with flag-tagged dynein heavy chain constructs using Fugene (Promega). Cell extracts were prepared by lysing cells on ice in BRB80 (80 mM PIPES, $1 \mathrm{mM} \mathrm{MgCl} 2,1 \mathrm{mM}$ EGTA, pH 6.8) supplemented with $1 \%$ Triton-X, protease inhibitors (AEBSF, Aprotinin, Bestatin, E64 and Leupeptin), and $1 \mathrm{mM} \mathrm{MgATP}$. Cell extract was centrifuged at $2,000 \mathrm{~g}$ for $5 \mathrm{~min}$ and $20,000 \mathrm{~g}$ for $30 \mathrm{~min}$. The supernatant was frozen in LN2 for further use. Anti-Flag coated beads $(1.0 \mu \mathrm{m}$, carboxylated, Invitrogen) were incubated in cell extract for $60 \mathrm{~min}$ on ice, and then pelleted and resuspended in BRB80 two times before use.

Beads were diluted in motility buffer (BRB80 supplemented with $0.2 \mathrm{mg} \mathrm{ml}^{-1}$

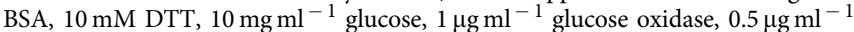
catalase, $2 \mathrm{mM}$ ATP and $20 \mu \mathrm{M}$ taxol) and added to a flow chamber. An optical trap was used to position beads over coverslip-attached MTs, and measure their forces and displacements using back-focal plane interferometry.

DNA constructs and microinjection. Expression clones were made in the pSM vector, a derivative of pPD49.26 (A. Fire) with extra cloning sites (S. McCarroll and C.I. Bargmann, personal communication). Plasmids and sequences are available upon request. All constructs were sequenced prior to microinjection. TagRFP optimized for expression in worms was a gift from C. Frøkjaer-Jensen. The Rat DHC1::FLAG was a gift from Dr R. Vallee. Microinjection was carried out using standard procedures. For each injected construct, at least 2-3 lines were generated to control for variation in expression levels. myrGFP::dhc-1 was constructed by 
recombineering a kanamycin_Pmig-13::myrGFP into WRM0639aB10, a dhc-1 containing fosmid. Pmig-13::LoxP::STOP::LoxP::TagRFP::PTRN-1a(cDNA) was generated using Gibson cloning. Pitr-1::DLC-1::GFP and Pitr-1::3XGFP::EMTB were generated by standard restriction cloning. The SMA mutation was cloned into Rat DHC1::FLAG using mutated primers to amplify a $\sim 3.5 \mathrm{~kb}$ fragment centred around the mutation, which was then cloned back into the vector with FseI.

Data availability. All strains and plasmids and data generated in this study are available from the authors.

\section{References}

1. Conde, C. \& Caceres, A. Microtubule assembly, organization and dynamics in axons and dendrites. Nat. Rev. Neurosci. 10, 319-332 (2009).

2. Janke, C. \& Kneussel, M. Tubulin post-translational modifications: encoding functions on the neuronal microtubule cytoskeleton. Trends Neurosci. 33, 362-372 (2010).

3. Kapitein, L. C. \& Hoogenraad, C. C. Building the neuronal microtubule cytoskeleton. Neuron 87, 492-506 (2015).

4. Coles, C. H. \& Bradke, F. Coordinating neuronal actin-microtubule dynamics. Curr. Biol. 25, R677-R691 (2015).

5. Millecamps, S. \& Julien, J. P. Axonal transport deficits and neurodegenerative diseases. Nat. Rev. Neurosci. 14, 161-176 (2013).

6. Tischfield, M. A., Cederquist, G. Y., Gupta, Jr M. L. \& Engle, E. C. Phenotypic spectrum of the tubulin-related disorders and functional implications of disease-causing mutations. Curr. Opin. Genet. Dev. 21, 286-294 (2011).

7. Desai, A. \& Mitchison, T. J. Microtubule polymerization dynamics. Annu. Rev. Cell Dev. Biol. 13, 83-117 (1997).

8. Ligon, L. A., Karki, S., Tokito, M. \& Holzbaur, E. L. Dynein binds to betacatenin and may tether microtubules at adherens junctions. Nat. Cell Biol. 3, 913-917 (2001).

9. Vinogradova, T., Miller, P. M. \& Kaverina, I. Microtubule network asymmetry in motile cells: role of Golgi-derived array. Cell Cycle 8, 2168-2174 (2009).

10. Zanic, M., Widlund, P. O., Hyman, A. A. \& Howard, J. Synergy between XMAP215 and EB1 increases microtubule growth rates to physiological levels. Nat. Cell Biol. 15, 688-693 (2013).

11. Baas, P. W. \& Black, M. M. Individual microtubules in the axon consist of domains that differ in both composition and stability. J. Cell Biol. 111, 495-509 (1990).

12. Brady, S. T., Tytell, M. \& Lasek, R. J. Axonal tubulin and axonal microtubules: biochemical evidence for cold stability. J. Cell Biol. 99, 1716-1724 (1984).

13. Baas, P. W., Ahmad, F. J., Pienkowski, T. P., Brown, A. \& Black, M. M. Sites of microtubule stabilization for the axon. J. Neurosci. 13, 2177-2185 (1993).

14. Li, Y. \& Black, M. M. Microtubule assembly and turnover in growing axons. J. Neurosci. 16, 531-544 (1996)

15. Sahenk, Z. \& Brady, S. T. Axonal tubulin and microtubules: morphologic evidence for stable regions on axonal microtubules. Cell Motil. Cytoskeleton 8, 155-164 (1987).

16. Hirokawa, N. Microtubule organization and dynamics dependent on microtubule-associated proteins. Curr. Opin. Cell Biol. 6, 74-81 (1994).

17. Song, Y. et al. Transglutaminase and polyamination of tubulin: posttranslational modification for stabilizing axonal microtubules. Neuron $\mathbf{7 8}$, 109-123 (2013).

18. Ghosh-Roy, A., Goncharov, A., Jin, Y. \& Chisholm, A. D. Kinesin-13 and tubulin posttranslational modifications regulate microtubule growth in axon regeneration. Dev. Cell 23, 716-728 (2012).

19. Yogev, S., Cooper, R., Fetter, R., Horowitz, M. \& Shen, K. Microtubule organization determines axonal transport dynamics. Neuron 92, 449-460 (2016).

20. Harada, A., Teng, J., Takei, Y., Oguchi, K. \& Hirokawa, N. MAP2 is required for dendrite elongation, PKA anchoring in dendrites, and proper PKA signal transduction. J. Cell Biol. 158, 541-549 (2002).

21. Teng, J. et al. Synergistic effects of MAP2 and MAP1B knockout in neuronal migration, dendritic outgrowth, and microtubule organization. J. Cell Biol. 155, 65-76 (2001).

22. Kardon, J. R. \& Vale, R. D. Regulators of the cytoplasmic dynein motor. Nat. Rev. 10, 854-865 (2009).

23. Vallee, R. B., McKenney, R. J. \& Ori-McKenney, K. M. Multiple modes of cytoplasmic dynein regulation. Nat. Cell Biol. 14, 224-230 (2012).

24. Bhabha, G., Johnson, G. T., Schroeder, C. M. \& Vale, R. D. How dynein moves along microtubules. Trends Biochem. Sci. 41, 94-105 (2016).

25. Maeder, C. I., Shen, K. \& Hoogenraad, C. C. Axon and dendritic trafficking. Curr. Opin. Neurobiol. 27, 165-170 (2014).

26. Maday, S., Twelvetrees, A. E., Moughamian, A. J. \& Holzbaur, E. L. Axonal transport: cargo-specific mechanisms of motility and regulation. Neuron 84, 292-309 (2014)

27. Koushika, S. P. et al. Mutations in Caenorhabditis elegans cytoplasmic dynein components reveal specificity of neuronal retrograde cargo. J. Neurosci. 24, 3907-3916 (2004).
28. Lloyd, T. E. et al. The p150(Glued) CAP-Gly domain regulates initiation of retrograde transport at synaptic termini. Neuron 74, 344-360 (2012).

29. Moughamian, A. J. \& Holzbaur, E. L. Dynactin is required for transport initiation from the distal axon. Neuron 74, 331-343 (2012).

30. Moughamian, A. J. \& Holzbaur, E. L. F. in Dyneins (ed. Stephen, M. King) 584-601 (Academic Press, 2012).

31. Lipka, J., Kuijpers, M., Jaworski, J. \& Hoogenraad, C. C. Mutations in cytoplasmic dynein and its regulators cause malformations of cortical development and neurodegenerative diseases. Biochem. Soc. Trans. 41, 1605-1612 (2013).

32. Scoto, M. et al. Novel mutations expand the clinical spectrum of DYNC1H1-associated spinal muscular atrophy. Neurology 84, 668-679 (2015).

33. Strickland, A. V. et al. Mutation screen reveals novel variants and expands the phenotypes associated with DYNC1H1. J. Neurol. 262, 2124-2134 (2015).

34. Harms, M. B. et al. Mutations in the tail domain of DYNC1H1 cause dominant spinal muscular atrophy. Neurology 78, 1714-1720 (2012).

35. Ahmad, F. J. et al. Motor proteins regulate force interactions between microtubules and microfilaments in the axon. Nat. Cell Biol. 2, 276-280 (2000)

36. Ahmad, F. J., Echeverri, C. J., Vallee, R. B. \& Baas, P. W. Cytoplasmic dynein and dynactin are required for the transport of microtubules into the axon. J. Cell Biol. 140, 391-401 (1998).

37. Baas, P. W., Vidya Nadar, C. \& Myers, K. A. Axonal transport of microtubules: the long and short of it. Traffic 7, 490-498 (2006).

38. Del Castillo, U., Winding, M., Lu, W. \& Gelfand, V. I. Interplay between kinesin-1 and cortical dynein during axonal outgrowth and microtubule organization in Drosophila neurons. eLife 4, e10140 (2015).

39. Dujardin, D. L. \& Vallee, R. B. Dynein at the cortex. Curr. Opin. Cell Biol. 14, 44-49 (2002).

40. McNally, F. J. Mechanisms of spindle positioning. J. Cell Biol. 200, 131-140 (2013).

41. Perlson, E. et al. Dynein interacts with the neural cell adhesion molecule (NCAM180) to tether dynamic microtubules and maintain synaptic density in cortical neurons. J. Biol. Chem. 288, 27812-27824 (2013).

42. Schmoranzer, J. et al. Par3 and dynein associate to regulate local microtubule dynamics and centrosome orientation during migration. Curr. Biol. 19, 1065-1074 (2009).

43. Hendricks, A. G. et al. Dynein tethers and stabilizes dynamic microtubule plus ends. Curr. Biol. 22, 632-637 (2012).

44. Laan, L. et al. Cortical dynein controls microtubule dynamics to generate pulling forces that position microtubule asters. Cell 148, 502-514 (2012).

45. Maeder, C. I., San-Miguel, A., Wu, E. Y., Lu, H. \& Shen, K. In vivo neuron-wide analysis of synaptic vesicle precursor trafficking. Traffic 15, 273-291 (2014).

46. Ou, C. Y. et al. Two cyclin-dependent kinase pathways are essential for polarized trafficking of presynaptic components. Cell 141, 846-858 (2010).

47. Arribere, J. A. et al. Efficient marker-free recovery of custom genetic modifications with CRISPR/Cas9 in Caenorhabditis elegans. Genetics 198, 837-846 (2014).

48. Hafezparast, M. et al. Mutations in dynein link motor neuron degeneration to defects in retrograde transport. Science 300, 808-812 (2003).

49. Ori-McKenney, K. M., Xu, J., Gross, S. P. \& Vallee, R. B. A cytoplasmic dynein tail mutation impairs motor processivity. Nat. Cell Biol. 12, 1228-1234 (2010)

50. Mazumdar, M., Mikami, A., Gee, M. A. \& Vallee, R. B. In vitro motility from recombinant dynein heavy chain. Proc. Natl Acad. Sci. USA 93, 6552-6556 (1996).

51. Hamill, D. R., Severson, A. F., Carter, J. C. \& Bowerman, B. Centrosome maturation and mitotic spindle assembly in C. elegans require SPD-5, a protein with multiple coiled-coil domains. Dev. Cell 3, 673-684 (2002).

52. Nguyen-Ngoc, T., Afshar, K. \& Gonczy, P. Coupling of cortical dynein and G alpha proteins mediates spindle positioning in Caenorhabditis elegans. Nat. Cell Biol. 9, 1294-1302 (2007).

53. O'Rourke, S. M., Dorfman, M. D., Carter, J. C. \& Bowerman, B. Dynein modifiers in C. elegans: light chains suppress conditional heavy chain mutants. PLoS Genet. 3, el28 (2007).

54. Yan, J. et al. Kinesin-1 regulates dendrite microtubule polarity in Caenorhabditis elegans. eLife 2, e00133 (2013).

55. Zheng, Y. et al. Dynein is required for polarized dendritic transport and uniform microtubule orientation in axons. Nat. Cell Biol. 10, 1172-1180 (2008).

56. Adler, C. E., Fetter, R. D. \& Bargmann, C. I. UNC-6/Netrin induces neuronal asymmetry and defines the site of axon formation. Nat. Neurosci. 9, 511-518 (2006).

57. Cooper, R., Yogev, S., Shen, K. \& Horowitz, M. MTQuant: 'Seeing' beyond the diffraction limit in fluorescence images to quantify neuronal microtubule organization. Preprint at bioRxiv https://doi.org/10.1101/074047 (2016).

58. Dillman, 3rd J. F., Dabney, L. P. \& Pfister, K. K. Cytoplasmic dynein is associated with slow axonal transport. Proc. Natl Acad. Sci. USA 93, 141-144 (1996).

59. Twelvetrees, A. E. et al. The dynamic localization of cytoplasmic dynein in neurons is driven by kinesin-1. Neuron 90, 1000-1015 (2016). 
60. Stepanova, T. et al. Visualization of microtubule growth in cultured neurons via the use of EB3-GFP (end-binding protein 3-green fluorescent protein). J. Neurosci. 23, 2655-2664 (2003).

61. Chen, L. et al. Axon regeneration pathways identified by systematic genetic screening in C. elegans. Neuron 71, 1043-1057 (2011).

62. Richardson, C. E. et al. PTRN-1, a microtubule minus end-binding CAMSAP homolog, promotes microtubule function in Caenorhabditis elegans neurons. eLife 3, e01498 (2014).

63. Marcette, J. D., Chen, J. J. \& Nonet, M. L. The Caenorhabditis elegans microtubule minus-end binding homolog PTRN-1 stabilizes synapses and neurites. eLife 3, e01637 (2014).

64. Wang, S. et al. NOCA-1 functions with gamma-tubulin and in parallel to Patronin to assemble non-centrosomal microtubule arrays in C. elegans. eLife 4, e08649 (2015).

65. Chen, L., Stone, M. C., Tao, J. \& Rolls, M. M. Axon injury and stress trigger a microtubule-based neuroprotective pathway. Proc. Natl Acad. Sci. USA 109, 11842-11847 (2012).

66. Janson, M. E., de Dood, M. E. \& Dogterom, M. Dynamic instability of microtubules is regulated by force. J. Cell Biol. 161, 1029-1034 (2003).

67. Jung, M. K., Wilder, I. B. \& Oakley, B. R. Amino acid alterations in the benA (beta-tubulin) gene of Aspergillus nidulans that confer benomyl resistance. Cell Motil. Cytoskeleton 22, 170-174 (1992).

68. Wright, A. J. \& Hunter, C. P. Mutations in a beta-tubulin disrupt spindle orientation and microtubule dynamics in the early Caenorhabditis elegans embryo. Mol. Biol. Cell 14, 4512-4525 (2003).

\section{Acknowledgements}

We are thankful to C. Gao for technical assistance and to members of the Shen lab for fruitful discussions. We are also grateful to Dr R. Vallee for the Rat DHC1-FLAG plasmid and to Abdullah R. Chaudhary for DNA preparation and transfections. This work was funded by HHMI, R01NS082208, R01 NS034307 to K.S. and HSFP fellowship to S.Y.

\section{Author contributions}

K.S. and S.Y. designed the study, interpreted the results and wrote the manuscript S.Y. performed most of the experiments. A.G.H. conducted the force measurements on purified dynein. C.I.M. performed and analysed live imaging shown in Supplementary Fig. 3. R.C. and M.H. analysed data for Supplementary Fig. 5.

\section{Additional information}

Supplementary Information accompanies this paper at http://www.nature.com/ naturecommunications

Competing interests: The authors declare no competing financial interests.

Reprints and permission information is available online at http://npg.nature.com/ reprintsandpermissions/

How to cite this article: Yogev, S. et al. Local inhibition of microtubule dynamics by dynein is required for neuronal cargo distribution. Nat. Commun. 8, 15063 doi: 10.1038/ncomms15063 (2017).

Publisher's note: Springer Nature remains neutral with regard to jurisdictional claims in published maps and institutional affiliations.

(c) (i) article are included in the article's Creative Commons license, unless indicated otherwise in the credit line; if the material is not included under the Creative Commons license, users will need to obtain permission from the license holder to reproduce the material. To view a copy of this license, visit http://creativecommons.org/licenses/by/4.0/

(C) The Author(s) 2017 